\title{
Power spectrum of nuclear spectra with missing levels and mixed symmetries
}

\author{
R.A. Molina ${ }^{\mathrm{a}, \mathrm{c}, *}$, J. Retamosa $^{\mathrm{b}}$, L. Muñoz $^{\mathrm{b}}$, A. Relaño $^{\mathrm{b}, \mathrm{c}}$, E. Faleiro $^{\mathrm{d}}$ \\ a Max-Planck-Institut für Physik Komplexer Systeme, Nöthnitzer Str. 38, D-01187 Dresden, Germany \\ ${ }^{\mathrm{b}}$ Departamento de Física Atómica, Molecular y Nuclear, Universidad Complutense de Madrid, E-28040 Madrid, Spain \\ c Instituto de Estructura de la Materia, CSIC, Serrano 123, E-28006 Madrid, Spain \\ d Departamento de Física Aplicada, Universidad Politécnica de Madrid, E-28012, Spain
}

Received 22 June 2006; received in revised form 11 October 2006; accepted 26 October 2006

Available online 13 November 2006

Editor: J.-P. Blaizot

\begin{abstract}
Sequences of energy levels in nuclei are often plagued with missing levels whose number and position are unknown. It is also quite usual that all the quantum numbers of certain levels cannot be experimentally determined, and thus levels of different symmetries are mixed in the same sequence. The analysis of these imperfect spectra (from the point of view of spectral statistics) is unavoidable if one wants to extract some statistical information. The power spectrum of the $\delta_{q}$ statistic has emerged in recent years as an important tool for the study of quantum chaos and spectral statistics. We derive analytical expressions for the observed power spectrum in terms of the fraction of observed levels and the number of mixed sequences. These expressions are tested with large shell model spectra simulating realistic experimental situations. A good estimation of the number of mixed symmetries and the fraction of missing levels is obtained by means of a least-squares fit in a wide set of different situations. (c) 2006 Elsevier B.V. All rights reserved.
\end{abstract}

PACS: 21.60.-k; 05.45.Mt; 24.60.Lz

Keywords: Statistical spectroscopy; Quantum chaos; Shell model

\section{Introduction}

Spectral statistical analysis has become the main tool for the study of quantum chaos. It is now well established, through numerical simulations, theoretical studies using the semiclassical approximation, and the analysis of experimental data, that the spectral statistics of systems whose classical analog is chaotic follow the predictions of random matrix theory (RMT). When the classical analog is regular, the statistical properties of the spectra are identical to the properties of a sequence of uncorrelated random numbers and follow Poisson statistics [1]. Most effort has gone to study simple systems with a few degrees of freedom like quantum billiards (and their experimental realizations), the hydrogen atom in a strong mag-

\footnotetext{
* Corresponding author.

E-mail address: molina@iem.cfmac.csic.es (R.A. Molina).
}

netic field, etc. However, it is not clear whether the underlying classical regular or chaotic dynamics is the universal origin of the observed spectral fluctuations, since there exist systems without a classical analog that display RMT spectral statistics.

Actually, RMT was originally devised for the analysis of spectra of complex nuclei. Beyond the neutron emission threshold, the statistical properties of compound nucleus resonances with fixed quantum numbers were shown to follow the predictions of the Gaussian orthogonal ensemble (GOE) [2]. However, in the region closer to the ground state the situation is still unclear because of the experimental difficulty in obtaining complete sequences of levels. Even if data coming from different nuclei are properly studied, the results are less than conclusive [3-5]. More work is needed to completely understand the statistical properties of low-energy nuclear spectra.

In traditional random matrix studies, the level sequences are assumed to be complete and involving only one symmetry. Ran- 
dom matrix results apply only to cases where these conditions are fulfilled. However, experimental data cannot always guarantee these conditions. The change in the spectral properties due to the superposition of different level sequences or to the existence of missing levels has been studied since long ago. In [6] the authors give general expressions for the k-point cluster functions, and results for the number variance statistic $\Sigma^{2}$ were already reported in [7]. Also, the next-neighbor spacing distribution $P(s)$ was studied when different symmetries are mixed in the spectra [8,9]; the long-range correlations for this case were derived by Guhr et al. in their review about RMT [10]. Very recently, Bohigas and Pato used the ideas of Ref. [6] to study how the next-neighbor spacing distribution $P(s)$ behaves when only a fraction $\varphi$ of the levels is detected: they were able to give some interpolating formulas between the Wigner surmise and the Poisson distribution as a function of $\varphi$ [11]. It is worth commenting that their expression was already proposed as an ansatz in Ref. [12], and derived later by using information theory in Ref. [13]. The expression derived in these papers has been successfully applied in several cases $[13,14]$.

A new approach to energy level fluctuations based on methods from time series analysis has been proposed recently [15]. The $\delta_{q}$ statistic is viewed as a time series with the index $q$, that represents the order in energy, playing the role of a pseudotime. Analyzing the power spectrum of this statistic, it has been shown that chaotic quantum systems are characterized by $1 / f$ noise and integrable quantum systems by $1 / f^{2}$ noise. The full functional forms of the power spectrum were derived for the RMT ensembles [16]. The analysis of this new statistic has been shown to be extremely useful in different situations by a number of authors [17-23].

It is the purpose of this Letter to analyze the power spectrum of the $\delta_{q}$ statistic for sequences with missing levels and mixed symmetries. We will derive analytical formulas that will show the transition between a RMT power spectrum and a Poisson spectrum as a function of $\varphi$ and the number $l$ of mixed symmetries. We will show that through a fit to these formulas it is possible to estimate a value of $\varphi$ and $l$ from experimental data. To demonstrate the applicability of our results, we will study realistic nuclear spectra simulated through shell model calculations.

\section{Statistical analysis of spectra with missing levels and mixed symmetries}

The objective of every spectral statistical analysis is to characterize the properties of the fluctuations of the spectra. In order to extract the information contained in the spectral fluctuations, we have to separate them from the secular behavior of the level density. We assume that the density of states $g(E)$ can be separated into a smooth part and a fluctuating part,

$g(E)=\bar{g}(E)+\tilde{g}(E)$,

where $\bar{g}(E)$ is the smooth part of the level density and $\tilde{g}(E)$ is the fluctuating part. The accumulated level density $N(E)$ measures the number of levels up to a certain energy $E$ in the system. Thus it is related to the level density through

$$
N(E)=\int_{-\infty}^{E} d E^{\prime} g\left(E^{\prime}\right)
$$

and obviously it can also be separated into a smooth and a fluctuating part,

$N(E)=\bar{N}(E)+\tilde{N}(E)$.

The first step for doing the spectral statistical analysis is the proper unfolding of the data with the smooth level density, in order to be able to extract the fluctuations and compare them to the results of RMT. Using the mean accumulated level density $\bar{N}(E)$, the unfolded spectra $\left\{\epsilon_{i}\right\}$ can be obtained from the experimental spectra $\left\{E_{i}\right\}$ as $\epsilon_{i}=\bar{N}\left(E_{i}\right)$. Once the unfolding is performed, the level density becomes

$\rho(\epsilon)=\frac{g(E)}{\bar{g}(E)}=1+\frac{\tilde{g}(E)}{\bar{g}(E)}=1+\tilde{\rho}(\epsilon)$.

It can also be separated into a smooth constant part $\bar{\rho}(\epsilon)=1$ and a fluctuating part $\tilde{\rho}(\epsilon)$. The latter defines the fluctuating part of the accumulated density

$\tilde{n}(\epsilon)=\int_{-\infty}^{\epsilon} d \zeta \tilde{\rho}(\zeta)$

which satisfies that $\tilde{n}(\epsilon)=\tilde{N}(E)$ with $\epsilon=\bar{N}(E)$.

The $\delta_{q}$ statistic is defined using the unfolded energies as

$\delta_{q}=\epsilon_{q+1}-\epsilon_{1}-q$,

and it represents the deviation of the excitation energy of the $(q+1)$ th unfolded level from its mean value. Moreover, if we appropriately shift the ground state of the system, we can write

$\delta_{q}=-\tilde{n}\left(\epsilon_{q+1}\right)$,

that is, the accumulated level density fluctuations at $\epsilon=\epsilon_{q+1}$. We consider the $\delta_{q}$ function as a discrete time series, where the index $q$ plays the role of a pseudo-time, and analyze their properties with numerical techniques normally used in the study of complex systems. The most simple technique is the calculation of the power spectrum

$P_{k}^{\delta}=\frac{1}{N}\left|\sum_{q=0}^{N-1} \delta_{q} \exp (-2 \pi q k / N)\right|^{2}$,

where $N$ is the total number of levels in the sequence.

In this Letter, we will use RMT to derive theoretical expressions for the $\delta_{q}$ power spectrum when only a fraction $\varphi$ of levels is observed, or there are several sequences mixed. The three canonical random matrix ensembles are considered as the paradigmatic models of chaotic systems. They differ in the symmetry group under which they are invariant [9]: the Gaussian orthogonal ensemble (GOE) corresponds to systems with timereversal symmetry and spin-rotation symmetry; the Gaussian unitary ensemble (GUE) applies when the system has no timereversal symmetry, and finally, systems with time-reversal symmetry but no spin rotation symmetry are well described by 
the Gaussian symplectic ensemble (GSE). Their spectra display level correlations at all scales. Short range correlations are usually measured by means of the nearest neighbor spacing distribution $P(s)$. It behaves as $P(s) \propto s^{\beta}$ when $s \ll 1$ for the three canonical ensembles. This means that the probability of finding two neighboring levels at a distance $s$ is proportional to $s^{\beta}$, provided that $s$ is small enough. Therefore, the exponent $\beta$ measures the "repulsion" between consecutive levels. The repulsion parameter is $\beta=1,2,4$ for GOE, GUE and GSE, respectively. On the contrary, there is no level repulsion in integrable systems since their energy levels are uncorrelated.

Since the nucleon-nucleon interaction is time-reversal invariant, GOE is the appropriate ensemble to study the fluctuation properties of nuclei. Nevertheless, we will write general expressions for the three classical ensembles, and compare the shell model results with the GOE predictions.

It has been shown [16] that the ensemble average of the $\delta_{q}$ power spectrum $\left\langle P_{k}^{\delta}\right\rangle$ can be written in a compact form as

$$
\begin{aligned}
\left\langle P_{k}^{\delta}\right\rangle= & \frac{N^{2}}{4 \pi^{2}}\left[\frac{K(k / N)-1}{k^{2}}+\frac{K(1-k / N)-1}{(N-k)^{2}}\right] \\
& +\frac{1}{4 \sin ^{2}\left(\frac{\pi k}{N}\right)}+\Delta,
\end{aligned}
$$

valid for $N \gg 1, k \in[1,2, \ldots, N-1]$ and $\beta=0,1,2$ and 4 , i.e., for the three canonical ensembles as well as for integrable systems. In the following we will assume that the statistical properties of the level sequences are well described by these type of ensembles and that the conditions for $N$ and $k$ are fulfilled.

The function $K(\tau)$ is the so-called spectral form factor

$$
K(\tau)=\left\langle\lim _{L \rightarrow \infty} \frac{1}{2 L}\left|\int_{-L}^{L} d \epsilon \tilde{\rho}(\epsilon) e^{-2 \pi i \epsilon \tau}\right|^{2}\right\rangle .
$$

Exact analytical expressions are known for $K(\tau)$ in GOE, GUE, GSE and regular systems [9]. Eq. (10) can be written as

$$
\begin{aligned}
K(\tau) & =\int_{-\infty}^{\infty} d \epsilon\left[\delta(\epsilon)-1+R_{2}(\epsilon)\right] \exp (-2 \pi i \epsilon \tau) \\
& =1-\int_{-\infty}^{\infty} d \epsilon Y_{2}(\epsilon) \exp (-2 \pi i \epsilon),
\end{aligned}
$$

where $R_{2}$ and $Y_{2}$ are the two-point correlation function and the two-point cluster function, respectively. The $n$-point correlation function $R_{n}\left(\epsilon_{1}, \ldots, \epsilon_{n}\right)$ (see Ref. [9] for details) is the probability density of finding $n$ levels (without considering their labels) around the positions $\epsilon_{1}, \epsilon_{2}, \ldots, \epsilon_{n}$, the positions of the remaining levels unobserved. The cluster functions $Y_{n}\left(\epsilon_{1}, \ldots, \epsilon_{n}\right)$ describe the clustering of the $n(>1)$ levels in small subgroups. Since we are considering unfolded spectra that become translationally invariant in the limit $N \rightarrow \infty$, the functions $R_{n}$ and $Y_{n}$ turn out to depend only on the differences $\left|\epsilon_{i}-\epsilon_{j}\right|$. Therefore we can write $R_{2}\left(\epsilon_{1}, \epsilon_{2}\right)=R_{2}\left(\epsilon_{2}-\epsilon_{1}\right)=R_{2}(\epsilon)$ and $Y_{2}\left(\epsilon_{1}, \epsilon_{2}\right)=Y_{2}\left(\epsilon_{2}-\epsilon_{1}\right)=Y_{2}(\epsilon)$, which are the expressions appearing in Eq. (11).
The constant term $\Delta$ is a subtle consequence of the discrete nature of $\delta_{q}$. It depends on the difference between the variances of $\delta_{q}$ and $\left.\tilde{n}(\epsilon)\right|_{\epsilon=q}$, whose large $\mathrm{q}$ behavior is [7]

$$
\begin{aligned}
\left\langle\delta_{q}^{2}\right\rangle-\left\langle\tilde{n}(q)^{2}\right\rangle= & \frac{1}{3} \int_{-\infty}^{\infty} d r d s Y_{3}(0, r, s) \\
& -\frac{1}{2}\left(\int_{-\infty}^{\infty} d r Y_{2}(r)\right)^{2}, \quad q \gg 1,
\end{aligned}
$$

except in the rare cases where the integrals do not exist. The r.h.s. is zero for Poisson and $-1 / 6$ for the canonical ensembles. A straightforward calculation [16] allows us to obtain

$\Delta= \begin{cases}-\frac{1}{12} & \text { for canonical ensembles, } \\ 0 & \text { for Poisson. }\end{cases}$

Our main objective is to obtain a theoretical expression for the average power spectrum of an energy level sequence with missing levels and mixed symmetries. Suppose that there are $l$ complete, infinite and stationary level sequences with level densities $\rho_{i}(\epsilon)$, whose spectral fluctuations are given by an appropriate ensemble. Then, the crucial step is to find how the original spectral form factor $K(\tau)$ is modified when these sequences are superposed and some levels are dropped randomly from each sequence. The relevant parameters of the problem are the probability $\varphi_{i}\left(\epsilon_{q}\right)$ of observing one given level with energy $\epsilon_{q}$ pertaining to the $i$ th sequence, and the fractional densities $\eta_{i}=\overline{\rho_{i}}(\epsilon) /\left(\sum_{i=1}^{l} \overline{\rho_{i}}(\epsilon)\right)$. In order to make the statistical analysis tractable we consider two basic assumptions:

(1) The probabilities $\varphi_{i}\left(\epsilon_{q}\right)$ are set equal to a constant $\varphi_{i}$ for each sequence, meaning that the energy levels are dropped randomly and uniformly from the spectrum; from now on these quantities will be called the fractions of observed levels.

(2) The fractional densities are taken constant through the whole spectrum; therefore, except for a constant factor, the smooth shape of the different sequences is the same.

We shall consider first and in great detail the case of a single incomplete level sequence, which is most likely the situation most relevant to experiments in nuclear physics. Assuming that the fraction of observed levels is $0<\varphi<1$, it can be shown that the $n$-point cluster functions are modified as [11]

$\mathcal{Y}_{n}\left(\epsilon_{1}, \ldots, \epsilon_{n}\right)=Y_{n}\left(\epsilon_{1} / \varphi, \ldots, \epsilon_{n} / \varphi\right)$.

In what follows capital letters denote the statistical quantities of the actual spectrum, while "calligraphic" letters denote the same quantities for the observed spectrum. Then the two-point cluster function becomes

$\mathcal{Y}_{2}(\epsilon)=Y_{2}(\epsilon / \varphi)$

where we have already taken into account that it only depends on the energy difference $\epsilon=\epsilon_{1}-\epsilon_{2}$. Using the properties of the Fourier transform, it is immediate to see that

$\mathcal{K}(\tau)=1-\varphi+\varphi K(\varphi \tau)$. 
Since the correlations should approach those of a Poisson sequence as the number of missing levels increases, the constant term $\Delta$ is expected to reduce with $\varphi$. Introducing Eq. (14) into Eq. (12) one can easily see that

$\Delta \rightarrow \varphi^{2} \Delta$.

Collecting together formulas (16), (17) and (9) we arrive to the analytical expression for the average power spectrum of the $\delta_{q}$ statistic as a function of $\varphi$

$$
\begin{aligned}
\left\langle\mathcal{P}_{k}^{\delta}\right\rangle= & \frac{N^{2} \varphi}{4 \pi^{2}}\left[\frac{K\left(\frac{\varphi k}{N}\right)-1}{k^{2}}+\frac{K\left(\frac{\varphi(N-k)}{N}\right)-1}{(N-k)^{2}}\right] \\
& +\frac{1}{4 \sin ^{2}(\pi k / N)}-\varphi^{2} \Delta .
\end{aligned}
$$

In the case of a Poisson sequence, where the levels are uncorrelated, and the fluctuations must remain invariant as $\varphi$ decreases, $K(\tau)=1$ and $\Delta=0$ we obtain

$\left\langle\mathcal{P}_{k}^{\delta}\right\rangle_{\beta=0}=\frac{1}{4 \sin ^{2}\left(\frac{\pi k}{N}\right)}$,

for all $0<\varphi<1$. As it is expected, this Poisson limit is also reached when $\varphi \rightarrow 0$, regardless of the values of $K(\tau)$, i.e.,

$\left\langle\mathcal{P}_{k}^{\delta}\right\rangle \stackrel{\varphi \rightarrow 0}{\longrightarrow} \frac{1}{4 \sin ^{2}\left(\frac{\pi k}{N}\right)}$.

Let us now consider the superposition of $l$ level sequences with different quantum numbers and constant fractional densities $\eta_{i}$. It is easy to find how the two-level cluster function is modified in this case [10],

$\mathcal{Y}_{2}(\epsilon)=\sum_{i=1}^{l} \eta_{i}^{2} Y_{2, i}\left(\eta_{i} \epsilon\right)$.

If the sequences are incomplete the two-level cluster function can be written as

$\mathcal{Y}_{2}(\epsilon)=\sum_{i=1}^{l} \eta_{i}^{2} Y_{2, i}\left(\eta_{i} \epsilon / \varphi_{i}\right)$

where $\varphi_{i}$ is the fraction of observed levels in the $i$ th sequence.

Using Eq. (22) and going through the same steps that lead to Eq. (18), one obtains the average power spectrum in the general case

$$
\begin{aligned}
\left\langle\mathcal{P}_{k}^{\delta}\right\rangle= & \frac{N^{2}}{4 \pi^{2}} \sum_{i=1}^{l} \eta_{i} \varphi_{i}\left[\frac{K_{i}\left(\frac{\varphi_{i} k}{N \eta_{i}}\right)-1}{k^{2}}+\frac{K_{i}\left(\frac{\varphi_{i}(N-k)}{N \eta_{i}}\right)-1}{(N-k)^{2}}\right] \\
& +\frac{1}{4 \sin ^{2}(\pi k / N)}+\langle\varphi\rangle^{2} \Delta,
\end{aligned}
$$

where $K_{i}(\tau)$ is the spectral form factor characteristic of the $i$ th sequence and $\left\langle\varphi^{2}\right\rangle=\sum_{i=1}^{l} \eta_{i}\left(\varphi_{i}\right)^{2}$. Note that

$\left\langle\mathcal{P}_{k}^{\delta}\right\rangle_{\beta=0}=\frac{1}{4 \sin ^{2}\left(\frac{\pi k}{N}\right)}$,

i.e., the superposition of an arbitrary number of incomplete Poisson spectra gives rise to a new uncorrelated sequence of levels.

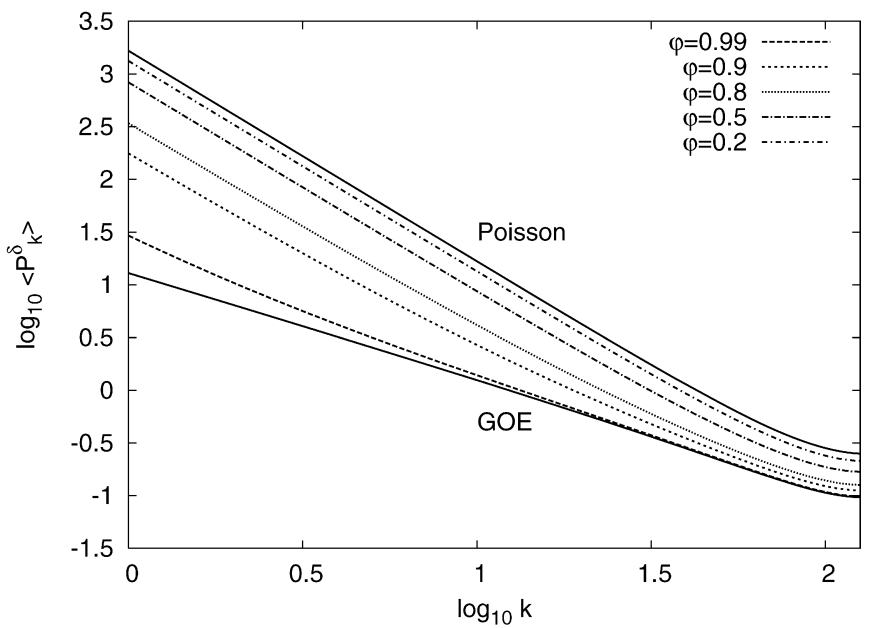

Fig. 1. Theoretical average power spectrum, predicted by Eq. (23), as a function of the fraction of observed levels $\varphi$ for pure sequences of length $N=256$. The curves are compared with the GOE and Poisson limits.

It is interesting to study the low frequency limit of this expression when several chaotic ( $\beta=1,2$ and 4$)$ level sequences are mixed. A Taylor expansion of Eq. (23) shows that its first term becomes dominant when $k \ll N$ and $N \gg 1$, so we can write

$\left\langle\mathcal{P}_{k}^{\delta}\right\rangle=\frac{N \sum_{i=1}^{l}\left(\varphi_{i}\right)^{2}}{2 \pi^{2} \beta k}+\frac{(1-\langle\varphi\rangle) N^{2}}{4 \pi^{2} k^{2}}$.

This expression shows that for small frequencies, the excitation energy fluctuations exhibit a linear combination of $1 / f$ and $1 / f^{2}$ noises. If $1-\langle\varphi\rangle \gg l / N$ the second term dominates and we have a $1 / f^{2}$ spectrum

$\left\langle\mathcal{P}_{k}^{\delta}\right\rangle=\frac{(1-\langle\varphi\rangle) N^{2}}{4 \pi^{2} k^{2}}$.

In this case we can extract the average fraction of missing levels from the multiplicative constant. When there are no missing levels, the resulting expression is

$\left\langle\mathcal{P}_{k}^{\delta}\right\rangle=\frac{N l}{2 \pi^{2} k \beta}$,

so we have a strict $1 / f$ and the proportionality constant gives the number of mixed sequences $l$, independently of the values of fractional densities $\eta_{i}$.

In order to better see how the theoretical expressions look like, it is interesting to plot them in some paradigmatic cases, as it is done in Figs. 1 and 2. In both cases, the scenarios discussed are similar to those studied in Section 3; nevertheless the figures pursue only to illustrate the qualitative behavior of Eq. (23) in different situations.

Fig. 1 displays the theoretical predictions of Eq. (23) for pure GOE sequences of length $N=256$ and different values of $\varphi$. We immediately realize that the correlations are very sensitive to the fraction of missing levels, and actually there is an important difference with the GOE limit already for $\varphi=0.99$. In agreement with the predictions of Eq. (26), $\left\langle\mathcal{P}_{k}^{\delta}\right\rangle$ is consistent with a $1 / f^{2}$ noise at low frequencies. We can also see a crossover from this behavior to the $1 / f$ noise behavior of 


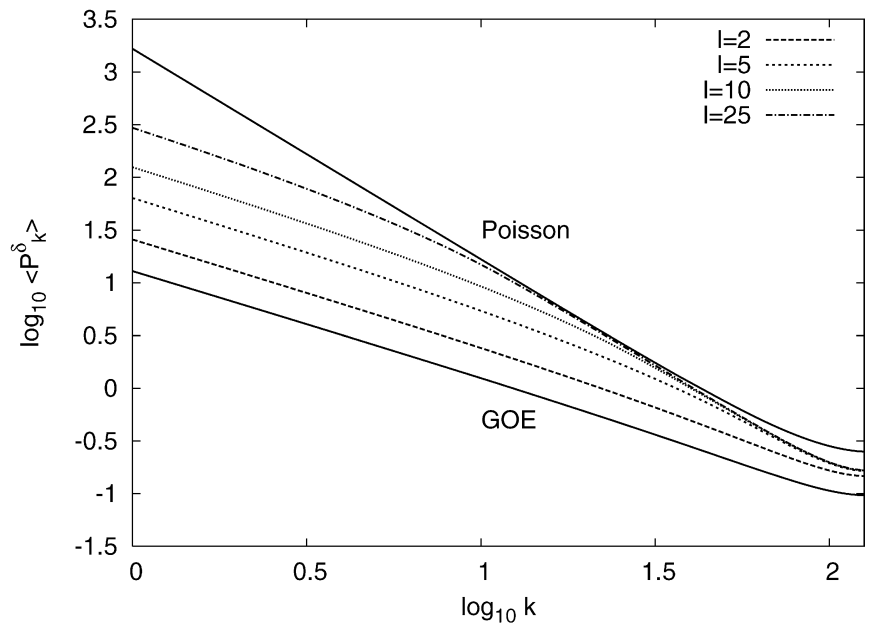

Fig. 2. Theoretical average power spectrum predicted by Eq. (23) for a mixed sequence obtained by superposing $l$ sequences of dimension $N=256$ and no missing levels. As in the previous figure we compare with the GOE and Poisson curves.

chaotic systems at large values of $k$. The $1 / f$ noise region gets smaller very quickly as $\varphi$ decreases, and for $\varphi \leqslant 0.8$ it is almost impossible to distinguish this part. However, the global factor of $\left\langle\mathcal{P}_{k}^{\delta}\right\rangle$ is very sensitive to small changes of $\varphi$ when this fraction is close to unity; therefore, the $\delta_{q}$ power spectrum analysis seems to be adequate to detect the existence of a small amount of missing levels by fitting the curve (26) to the experimental data. On the contrary, for $\varphi \approx 0$ the global factor changes very slowly and it is more difficult to obtain precise information on the fraction of observed levels.

Fig. 2 compares the predictions of the theoretical formula (23) for the mixing of $l$ pure GOE sequences of length $N=256$ with the GOE and Poisson limits. For simplicity, it has been assumed that all the fractional densities are equal to $\eta_{i}=1 / l$. In this case the fluctuations also exhibit a smooth transition from GOE to Poisson statistics, but the behavior of $\left\langle\mathcal{P}_{k}^{\delta}\right\rangle$ in the intermediate region is very different from the previous case. The theoretical curves (27) are parallel to the GOE limit up to a critical frequency $k_{c} \simeq N / l$; therefore, $\left\langle\mathcal{P}_{k}^{\delta}\right\rangle$ displays a $1 / f$ behavior in a wide frequency interval, even for the superposition of a large number of sequences.

\section{Analysis of shell model spectra}

To test if the theoretical expression (23) can be used to obtain a good estimation of the number of mixed symmetries and the fraction of observed levels, we have resorted to shell model calculations. After preparing several incomplete sequences, that may correspond to generic experimental situations, we have compared them to the predictions of a simplified version of Eq. (23) (see below for details). The values of the number of mixed symmetries and the fraction of observed levels are estimated by means of least-square fits. Moreover, this procedure has also allowed us to test some assumptions included in the calculations presented in Section 2 that may not be completely satisfied in experimental situations - the missing levels are dropped randomly and uniformly from the original spec-
Table 1

Dimensions of the $J(T=0)$ spectra of ${ }^{24} \mathrm{Mg}$ in the $s d$ shell

\begin{tabular}{l|lllllllll}
\hline$J$ & 0 & 1 & 2 & 3 & 4 & 5 & 6 & 7 & 8 \\
$N_{J}$ & 325 & 779 & 1206 & 1304 & 1311 & 1070 & 835 & 531 & 329 \\
\hline
\end{tabular}

trum, and the fractional level densities $\eta_{i}$ are constant through the whole spectrum.

As a representative example, we have considered the $s d$ nucleus ${ }^{24} \mathrm{Mg}$. This system was studied in Refs. [15,16] to show that the spectral fluctuations of quantum chaotic systems exhibit $1 / f$ noise. We follow the standard shell model procedure to obtain a large enough number of energy levels. The most bound particles are assumed to form an inert core while the remaining particles are allowed to move in a few single-particle levels, the so-called valence space. For ${ }^{24} \mathrm{Mg}$ it is usually considered an ${ }^{16} \mathrm{O}$ core and the valence space consists of the three shells $1 d^{5 / 2}, 2 s^{1 / 2}$ and $1 d^{3 / 2}$. The single particle energies and the two-body matrix elements correspond to the USD interaction of Wildenthal [24]. The construction and diagonalization of the $J T$ Hamiltonian matrices were carried out by using the shellmodel code NATHAN [25]. Because the valence space shells have positive parity all the states have positive parity. Moreover, the angular momentum and isospin quantum numbers can take the following values $J=0,1,2,3,4,5,6,7,8$ and $T=0,1,2$. The dimension of the $J(T=0)$ matrices, whose spectra have been used in the statistical analysis, are shown in Table 1.

Before we proceed to present the main results of this section, we describe briefly how the different types of sequences used in the statistical analysis are built:

(1) Mixed sequences. In order to obtain a mixed sequence, all the levels from different $J$ spectra are gathered together and ordered in increasing energy, regardless of their angular momentum; then, the resulting sequence is unfolded. Note that this method does not guarantee that the basic assumptions introduced in the theoretical calculations hold. The average $\delta_{q}$ power spectrum of the mixed sequence is calculated using a standard two-fold average procedure. The mixed sequence is divided in several sets of $N=256$ consecutive energy levels; the power spectrum of each set is calculated using a fast Fourier transform routine, and finally all these sets are used to compute some kind of "ensemble" average of the power spectrum. To improve this kind of average and clarify the main trend of the power spectrum, we divide the high frequency portion of the logarithmic frequency axis into equal bins and average locally the power spectrum components in each bin. Note that this last procedure can be performed if we have only a single sequence, for which the ensemble average is not possible.

(2) Pure sequences. After unfolding every $J$ spectrum, we divide it in several sets of $N=256$ consecutive levels. $\left\langle\mathcal{P}_{k}^{\delta}\right\rangle$ is calculated using the same procedure described above. First an ensemble average is carried out by averaging over the different sets of $N=256$ levels (notice that in this step we can use sets with different $J$ ), and then we perform the local average. 
Table 2

Results of fitting the power spectrum of different level sequences to Eq. (28). In the left side, "Fig." stands for the number of the figure where the results of the fit are presented; "mixing" can be equal to "pure" or "mixed" depending on the type of sequence we consider in each case; $J$ are the values of angular momentum involved in the calculation; and $\chi$ is the value of the cut-off or the average value of the cut-off function. In the right side we display the estimates of the two parameters appearing in Eq. (28): the fraction of observed levels $\varphi$ and the number of mixed sequences $l$

\begin{tabular}{llllll}
\hline Fig. & $J$ & Mixing & $\chi$ & $\varphi$ & $l$ \\
\hline 3 & 3,4 & Mixed & 1 & $1.00 \pm 0.01$ & $2.1 \pm 0.2$ \\
4 & $0-8$ & Mixed & 1 & $0.91 \pm 0.03$ & $7.2 \pm 0.6$ \\
5 & 3,4 & Mixed & 0.8 & $0.77 \pm 0.03$ & $2.1 \pm 0.4$ \\
5 & 3,4 & Pure & 0.8 & $0.80 \pm 0.03$ & $1.1 \pm 0.3$ \\
6 & $0-8$ & Mixed & 0.8 & $0.7 \pm 0.1$ & $11 \pm 3$ \\
6 & $0-8$ & Pure & 0.8 & $0.77 \pm 0.02$ & $0.9 \pm 0.1$ \\
7 & 3,4 & Mixed & $\langle\chi(\epsilon)\rangle=0.8$ & $0.73 \pm 0.03$ & $1.5 \pm 0.3$ \\
7 & 3,4 & Pure & $\langle\chi(\epsilon)\rangle=0.8$ & $0.82 \pm 0.02$ & $1.0 \pm 0.2$ \\
\hline
\end{tabular}

(3) Incomplete sequences. To generate incomplete sequences (pure or mixed) we first consider the complete and pure sequences that are going to be analyzed. Running along each sequence, the decision of keeping or dropping a given level $\epsilon_{q}$ is made by means of a random variable $x$ uniformly distributed in the interval $[0,1]$ and a smooth cut-off function $0 \leqslant \chi(\epsilon) \leqslant 1$ satisfying that $\sum_{q=1}^{N} \chi\left(\epsilon_{q}\right) / N=\varphi$. If $x>\chi\left(\epsilon_{q}\right)$ the level is dropped from the spectrum. With this procedure we drop roughly a fraction $(1-\varphi)$ of the levels, but note that this quantity can be slightly different from one sequence to another. For incomplete sequences, the unfolding is performed after all the levels have been dropped and the resulting sequences are mixed.

The expression (23) has too many free parameters to perform a meaningful fit; moreover, the number of mixing symmetries $l$ also determines the number of terms involved in the sum. Therefore, to obtain a realistic estimation of the most significant parameters, the number of mixing symmetries $l$ and the fraction of observed levels $\varphi$, a further simplification is needed. In what follows, we will consider the two following assumptions: (a) the fraction of observed levels is the same for all the mixed sequences $\varphi_{i}=\varphi$, and (b) the fractional densities are also the same $\eta_{i}=\eta=1 / l$. In such a case, the power spectrum of the $\delta_{n}$ function can be written as follows

$$
\begin{aligned}
\left\langle\mathcal{P}_{k}^{\delta}\right\rangle= & \frac{N^{2}}{4 \pi^{2}} \varphi\left[\frac{K\left(\frac{l \varphi k}{N}\right)-1}{k^{2}}+\frac{K\left(\frac{l \varphi(N-k)}{N}\right)-1}{(N-k)^{2}}\right] \\
& +\frac{1}{4 \sin ^{2}(\pi k / N)}+\varphi^{2} \Delta .
\end{aligned}
$$

This equation is going to be used to fit the power spectrum corresponding to different situations. In all the cases, the fit is performed by means of a nonlinear least-square algorithm, in logarithmic scale, and discarding the two first and the two last points. For the sake of clarity, we present the results in different figures, and present the values of the fitted parameters in Table 2.

Fig. 3 compares the average power spectrum of the $\delta_{q}$ function corresponding to the superposition of two level sequences

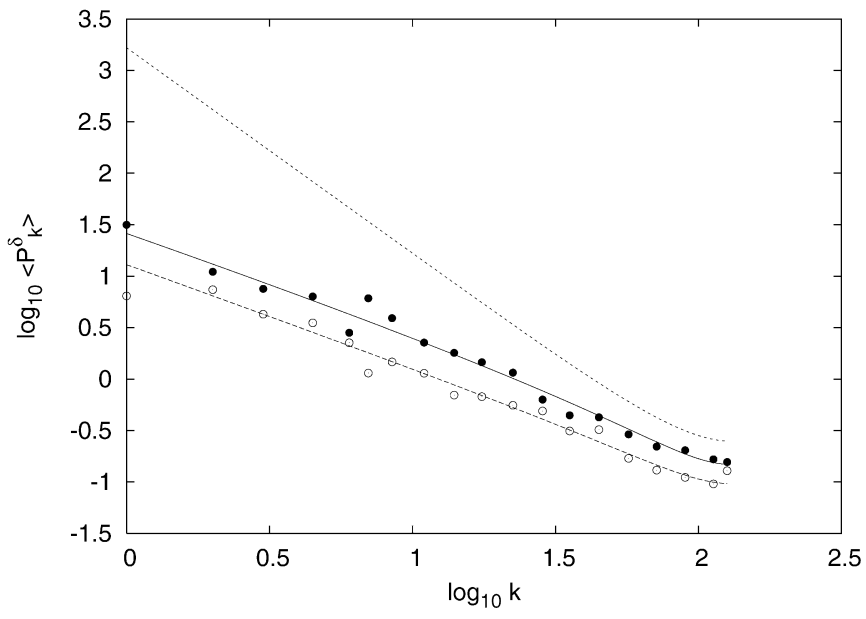

Fig. 3. Numerical $\left\langle\mathcal{P}_{k}^{\delta}\right\rangle$ calculated by using $J=3$ and $J=4$ pure sequences of length $N=256$ (open circles) and the superposition of these sequences (filled circles), compared to theoretical predictions: GOE (dashed line), Poisson (dotted line), and the result of fitting the mixed sequences to Eq. (28) (solid line).

with $J=3$ and $J=4$ to the fit of these data to (28), and with the theoretical predictions for the Poisson and GOE limits. Since the dimensions of the sequences are very similar, we have $\eta_{1} \simeq \eta_{2} \simeq 0.5$, and therefore the assumptions included in the derivation of (28) are essentially fulfilled. It can be seen that the agreement between the calculated points and the fitted curve is remarkable; the estimated parameters, included in the first row of Table 2, are in excellent agreement with the actual ones.

We can also consider a more involved situation where all the $J$ spectra of the $s d$ nucleus ${ }^{24} \mathrm{Mg}$ are taken into account. Therefore, we compute the average power spectrum of the $\delta_{q}$ statistic using nine pure sequences with $J=0,1, \ldots, 8$ and the superposition of all these sequences. The fractional densities are rather dissimilar in this case because the nine $J$ spectra have very different dimensions; thus deviations from the predictions of (28) are more probable. Fig. 4 compares $\left\langle P_{k}^{\delta}\right\rangle$ for the mixing of nine spectra with the fit of these data to Eq. (28) as well as with the theoretical predictions for the Poisson and GOE limits. We see that the fitted curve falls very close to the numerical points. Although the agreement between the estimated parameters and the values of $\varphi$ and $l$ is worse that in the previous case, their values are still quite reasonable and provide us a hint about the actual situation.

As commented above, experimental studies face the difficulty of establishing whether the level sequences are complete and how the missing levels distort the statistical properties. In order to get some insight on the effect of missing levels in the power spectrum of the $\delta_{q}$ function, we have generated incomplete sequences using the procedure described at the beginning of this section and a constant cut-off function $\chi(\epsilon)=0.8 \approx \varphi$. With this procedure we lose roughly $20 \%$ of the levels, but note that this quantity can be slightly different from one sequence to another, and therefore the basic assumptions of the theoretical calculation are not exactly fulfilled.

The average power spectrum of $\delta_{q}$ for two incomplete sequences with $J=3$ and $J=4$ and a mixed sequence is dis- 


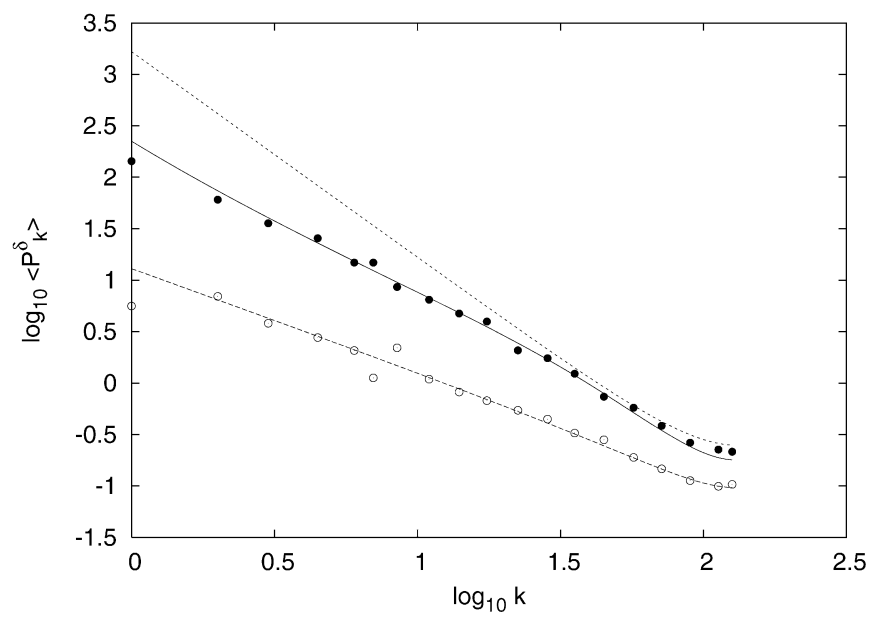

Fig. 4. Same as Fig. 3, but using nine different spectra with $J=0-8$.

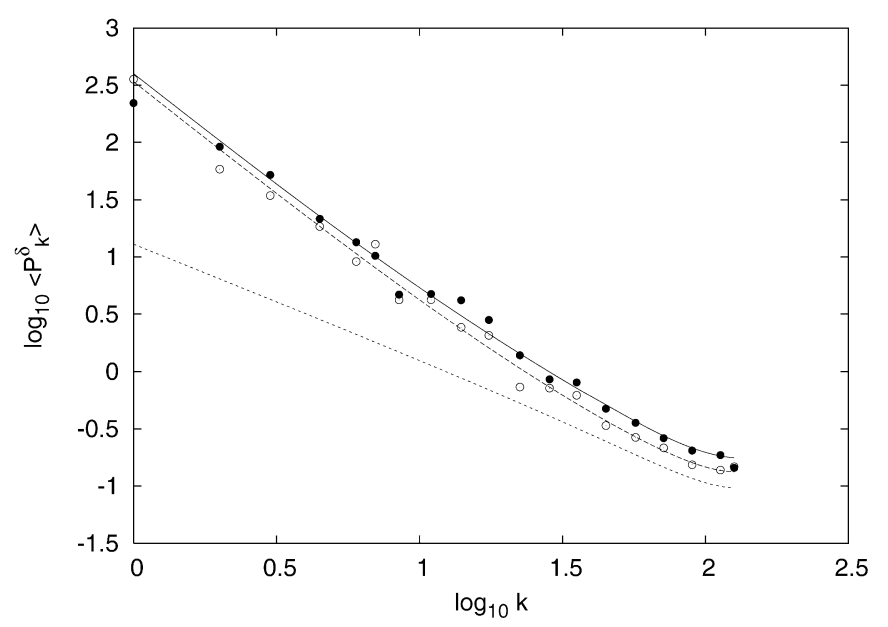

Fig. 5. Numerical $\left\langle\mathcal{P}_{k}^{\delta}\right\rangle$ calculated by using $J=3$ and $J=4$ incomplete pure sequences of length $N=256$ (open circles) and incomplete mixed sequences of the same length (filled circles), generated with a constant cut-off function $\chi=0.8$. These numerical power spectra are compared with fitted curves to Eq. (28): solid line corresponds to the fit of incomplete mixed sequences, and dashed line corresponds to fitting incomplete pure sequences. Theoretical predictions of Eq. (23) for GOE (dotted line) is also shown.

played in Fig. 5. The two power spectra, that are very close to the Poisson predictions, are indistinguishable for low $k$ values, and only the high frequency region seems to be sensitive to the mixing when there are missing levels. Nevertheless, a leastsquares fit to Eq. (28) makes it possible to distinguish between both situations and leads to an excellent estimation of the fraction of observed levels and of the number of mixed symmetries; the values of the fitted parameters can be found in the third and fourth rows of Table 2 .

Let us consider now a mixed (and incomplete) level sequence corresponding to all the $J$ spectra. In spite of the fact that the situation is quite involved, a least-squares fit to Eq. (28) gives rise to estimated parameters that are in striking agreement with the actual values of $\varphi$ and $l$ (see rows 5 and 6 of Table 2). Actually, the results of the fit do almost coincide with the exact parameters for pure sequences and are inside the error margin of the estimations when the different spectra are superposed.

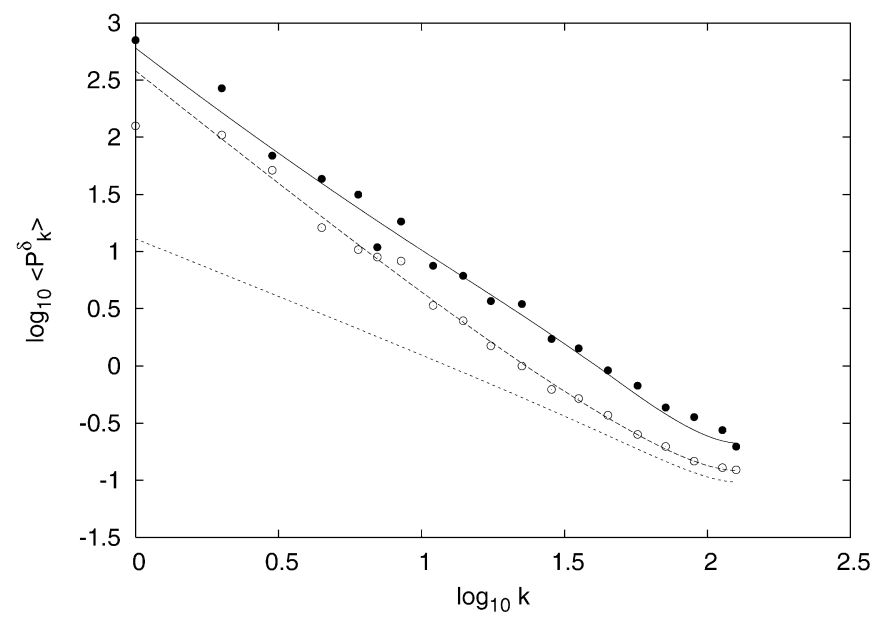

Fig. 6. Same as Fig. 5, but using all the calculated $J$ spectra of ${ }^{24} \mathrm{Mg}$.

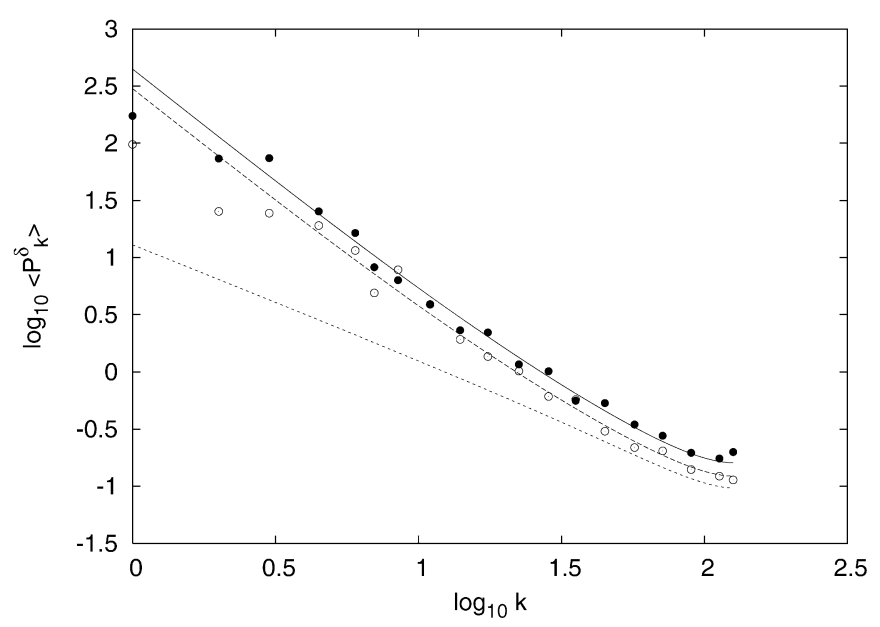

Fig. 7. Same as Fig. 5 for a cut-off function $\chi(\epsilon)$ that decreases linearly with the excitation energy.

Moreover, the fitted curves follow very closely the numerical values of $\left\langle P_{k}^{\delta}\right\rangle$, as it can be seen in Fig. 6 .

Up to this point, these examples suggest that the theoretical expressions derived in Section 2 may be a reasonable tool to obtain relevant information on the number of missing levels as well as on the number of mixed symmetries.

However, the assumption that energy levels are dropped at random and uniformly from the complete spectrum is probably not well justified. For this reason, we consider now a more realistic situation where the number of missing levels increase with the excitation energy. To test our theoretical expressions we use a cut-off function $\chi(\epsilon)$ that decreases smoothly with the energy, but keeping the average $\langle\chi(\epsilon)\rangle=0.8$. Fig. 7 shows the behavior of $\left\langle P_{k}^{\delta}\right\rangle$ for the incomplete $J=3$ and $J=4$ spectra as well as for the superposition of these two spectra; it also shows the two curves obtained by fitting the numerical points to (28). The plot looks like very similar to Fig. 5, except for the larger spreading of the numerical points at low $k$ values. In the case of pure level sequences, the energy dependence of $\chi$ does not spoil the results, since we obtain an excellent agreement for $l$ and $\varphi$ (row 8 of Table 2). For the mixed sequence the situation 
is clearly worse (see row 7 of Table 2). Even though the fitted value of $\varphi$ is slightly smaller than the actual one, the result is still reasonable; however the fit is not able to decide if there are two mixed sequences or if there is just only one pure sequence.

These results show that starting from the power spectrum of the $\delta_{q}$ function and the appropriate forms of the correlation functions, one can obtain a powerful tool to estimate the fraction of observed levels and the number of mixed symmetries in many experimental situations.

\section{Conclusions}

Using the $\delta_{q}$ function we have studied the distortion created by the existence of missing levels and mixed symmetries in the correlation structure of a given spectrum. We have derived theoretical expressions for the average power spectrum of the $\delta_{q}$ statistic; it depends on the fractions of observed levels $\varphi_{i}$ and the fractional densities $\eta_{i}$ of the different sequences that compose the spectrum. This theory predicts that the correlation structure of the spectrum undergoes a transition from the $1 / f$ noise of RMT spectra to the $1 / f^{2}$ noise characteristic of uncorrelated spectra as the fraction of missing levels or the number of mixed symmetries increase. One important result is that the intermediate stages of this transition are different in both cases: the low-frequency part of the power spectrum is very sensitive to the existence missing of levels, while the high-frequency part is more sensitive to the number of mixed sequences. Thus, if the statistical nature of the actual spectrum is known, it seems possible to extract information on the relevant parameters of the problem, by fitting the experimental power spectrum to the theoretical formulas. On the contrary, if the fraction of observed levels and the number of mixed sequences are known, we can obtain some hints about the actual correlation structure.

In order to test these expressions, we have used large scale shell model calculations. Complete $J T$ spectra have been appropriately transformed into imperfect spectra by dropping at random a fraction of levels or superposing several $J T$ spectra. Using a nonlinear least-square fit to the appropriate expression we have obtained estimates of the average fraction of observed levels $\langle\varphi\rangle$ as well as of the number of mixed symmetries $l$. In general, the agreement between the results of the fit and the actual parameters used to generate the level sequences ranges from good to remarkable. Thus we conclude that the method presented in this Letter is very useful to extract relevant statistical information from experimental spectra.

\section{Acknowledgements}

R.A.M. would like to thank E. Grosse, A. Wagner and G. Rusev of the Fz. Rossendorf for suggesting this work. A.R. is supported by the Spanish "Juan de la Cierva" program. This work is supported in part by Spanish Government grants BFM200304147-C02 and FTN2003-08337-C04-04.

\section{References}

[1] H.-J. Stöckmann, Quantum Chaos: An Introduction, Cambridge Univ. Press, Cambridge, 1999.

[2] R.U. Haq, A. Pandey, O. Bohigas, Phys. Rev. Lett. 48 (1982) 1086.

[3] G.E. Mitchell, E.G. Bilpuch, P.M. Endt, J.F. Shriner Jr., Phys. Rev. Lett. 61 (1988) 1473.

[4] J.F. Shriner Jr., E.G. Bilpuch, P.M. Endt, G.E. Mitchell, Z. Phys. A 335 (1990) 393.

[5] J.F. Shriner Jr., G.E. Mitchell, T. von Egidy, Z. Phys. A 338 (1991) 309.

[6] T.A. Brody, J. Flores, J.B. French, P.A. Mello, A. Pandey, S.S.M. Wong, Rev. Mod. Phys. 53 (1981) 385.

[7] J.B. French, V.K.B. Kota, A. Pandey, S. Tomsovic, Ann. Phys. (N.Y.) 181 (1988) 198.

[8] C.E. Porter, Statistical Theories of Spectra: Fluctuations, Academic Press, New York, 1965.

[9] M.L. Mehta, Random Matrices, Academic Press, New York, 1991.

[10] T. Guhr, A. Müller-Groeling, H.A. Weidenmüller, Phys. Rep. 299 (1998) 189.

[11] O. Bohigas, M.P. Pato, Phys. Lett. B 595 (2004) 171.

[12] W.A. Watson, E.G. Bilpuch, G.E. Mitchell, Nucl. Instrum. Methods 188 (1981) 571.

[13] U. Agvaanluvsan, G.E. Mitchell, J.F. Shriner Jr., M.P. Pato, Nucl. Instrum. Methods A 498 (2003) 459.

[14] U. Agvaanluvsan, G.E. Mitchell, J.F. Shriner Jr., M.P. Pato, Phys. Rev. C 67 (2003) 064608.

[15] A. Relaño, J.M.G. Gómez, R.A. Molina, J. Retamosa, E. Faleiro, Phys. Rev. Lett. 89 (2002) 244102.

[16] E. Faleiro, J.M.G. Gómez, R.A. Molina, L. Muñoz, A. Relaño, J. Retamosa, Phys. Rev. Lett. 93 (2004) 244101.

[17] A. Relaño, R.A. Molina, J. Retamosa, Phys. Rev. E 70 (2004) 017201.

[18] J.M.G. Gómez, A. Relaño, J. Retamosa, E. Faleiro, L. Salasnich, M. Vranicar, M. Robnik, Phys. Rev. Lett. 94 (2005) 084101.

[19] J. Barea, A. Frank, J.G. Hirsch, P. Van Isacker, Phys. Rev. Lett. 94 (2005) 102501.

[20] M.S. Santhanam, J.N. Bandyopadhyay, Phys. Rev. Lett. 95 (2005) 114101

[21] A. Relaño, J. Retamosa, E. Faleiro, R.A. Molina, A.P. Zuker, Phys. Rev. E 73 (2006) 026204.

[22] A.M. García García, Phys. Rev. E 73 (2006) 026213.

[23] E. Faleiro, U. Kuhl, R.A. Molina, L. Muñoz, A. Relaño, J. Retamosa, Phys. Lett. A 358 (2006) 251.

[24] B.H. Wildenthal, M.S. Curtin, B.A. Brown, Phys. Rev. C 28 (1983) 1343.

[25] E. Caurier, F. Nowacki, A. Poves, J. Retamosa, Phys. Rev. C 58 (1998) 2033. 